\title{
Target inhibition of galectin-3 by inhaled TD139 in patients with idiopathic pulmonary fibrosis
}

\author{
Nikhil Hirani ${ }^{1}$, Alison C. MacKinnon $\mathbb{1}^{1,2}{ }^{1}$, Lisa Nicol ${ }^{1}$, Paul Ford ${ }^{2}$, \\ Hans Schambye ${ }^{2}$, Anders Pedersen ${ }^{2}$, Ulf J. Nilsson ${ }^{3}$, Hakon Leffler $^{4}$, \\ Tariq Sethi ${ }^{2}$, Susan Tantawi ${ }^{2}$, Lise Gravelle $\mathbb{B}^{2}$, Robert J. Slack ${ }^{2}$, Ross Mills ${ }^{1}$, \\ Utsa Karmakar ${ }^{1}$, Duncan Humphries (i) ${ }^{1}$, Fredrik Zetterberg $\mathbb{(}^{2}$, Lucy Keeling ${ }^{5}$, \\ Lyn Paul ${ }^{6}$, Philip L. Molyneaux (10) ${ }^{6}$, Feng Li $\mathbb{1}^{1}$, Wendy Funston ${ }^{7}$, Ian A. Forrest ${ }^{8}$, \\ A. John Simpson (10,8, Michael A. Gibbons ${ }^{9}$ and Toby M. Maher ${ }^{6,10}$
}

Affiliations: ${ }^{1}$ MRC Centre for Inflammation Research, The Queen's Medical Research Institute, University of Edinburgh, Edinburgh, UK. ${ }^{2}$ Galecto, Copenhagen, Denmark. ${ }^{3}$ Dept of Chemistry, Lund University, Lund, Sweden. ${ }^{4}$ Dept of Laboratory Medicine, Lund University, Lund, Sweden. ${ }^{5}$ Exploristics, Belfast, UK. ${ }^{6}$ National Institute for Health Research Respiratory Clinical Research Facility, Royal Brompton and Harefield NHS Foundation Trust, and Fibrosis Research Group, National Heart and Lung Institute, Imperial College London, London, UK. ${ }^{7}$ Translational and Clinical Research Institute, Newcastle University, Newcastle upon Tyne, UK. ${ }^{8}$ Respiratory Medicine Unit, Royal Victoria Infirmary, Newcastle upon Tyne Hospitals NHS Foundation Trust, Newcastle upon Tyne, UK. ${ }^{9}$ Respiratory Dept, Institute of Biomedical and Clinical Science, Royal Devon and Exeter NHS Foundation Trust, Medical School, University of Exeter, Exeter, UK. ${ }^{10}$ Keck School of Medicine, University of Southern California, Los Angeles, CA, USA.

Correspondence: Nikhil Hirani, MRC Centre for Inflammation Research, The Queen's Medical Research Institute, University of Edinburgh, 47 Little France Crescent, Edinburgh, EH16 4TJ, UK. E-mail: n.hiranided.ac.uk

@ERSpublications

TD139 is a potent inhibitor of galectin-3, a key driver of fibrosis in the lung. In this phase 1/2a clinical study, inhaled TD139 was safe, well tolerated, and demonstrated target engagement and decreased plasma biomarkers associated with IPF progression. https://bit.ly/2JREKx6

Cite this article as: Hirani N, MacKinnon AC, Nicol L, et al. Target inhibition of galectin-3 by inhaled TD139 in patients with idiopathic pulmonary fibrosis. Eur Respir J 2021; 57: 2002559 [https://doi.org/ 10.1183/13993003.02559-2020].

ABSTRACT Galectin (Gal)-3 is a profibrotic $\beta$-galactoside-binding lectin that plays a key role in the pathogenesis of idiopathic pulmonary fibrosis (IPF) and IPF exacerbations. TD139 is a novel and potent small-molecule inhibitor of Gal-3.

A randomised, double-blind, multicentre, placebo-controlled, phase $1 / 2 \mathrm{a}$ study was conducted to assess the safety, tolerability, pharmacokinetics and pharmacodynamics of inhaled TD139 in 36 healthy subjects and 24 patients with IPF. Six dose cohorts of six healthy subjects were evaluated (4:2 TD139:placebo ratio) with single doses of TD139 $(0.15-50 \mathrm{mg})$ and three dose cohorts of eight patients with IPF (5:3 TD139: placebo ratio) with once-daily doses of TD139 (0.3-10 mg) for 14 days.

Inhaled TD139 was well tolerated with no significant treatment-related side-effects. TD139 was rapidly absorbed, with mean time taken to reach maximum plasma concentration $\left(C_{\max }\right)$ values ranging from 0.6 to $3 \mathrm{~h}$ and a plasma half-life $\left(T_{1 / 2}\right)$ of $8 \mathrm{~h}$. The concentration of TD139 in the lung was $>567$-fold higher than in the blood, with systemic exposure predicting exposure in the target compartment. Gal-3 expression on alveolar macrophages was reduced in the 3 and $10 \mathrm{mg}$ dose groups compared with placebo, with a concentration-dependent inhibition demonstrated. Inhibition of Gal-3 expression in the lung was associated with reductions in plasma biomarkers centrally relevant to IPF pathobiology (platelet-derived growth factor-BB, plasminogen activator inhibitor-1, Gal-3, CCL18 and YKL-40).

TD139 is safe and well tolerated in healthy subjects and IPF patients. It was shown to suppress Gal-3 expression on bronchoalveolar lavage macrophages and, in a concerted fashion, decrease plasma biomarkers associated with IPF progression. 


\section{Introduction}

Idiopathic pulmonary fibrosis (IPF) is a progressive, irreversible, ultimately fatal lung disease characterised by a progressive decline in lung function. The median survival is 3 years and incidence is increasing. The underlying pathogenesis of IPF is unknown; however, it most likely arises as a result of repeated alveolar epithelial cell injury and subsequent aberrant healing. It is considered likely that multiple intrinsic and environmental triggers might lead to IPF $[1,2]$. Two antifibrotic therapies have gained regulatory approval for use in IPF: pirfenidone, which has an uncertain mechanism of action, and nintedanib, which is a mixed tyrosine kinase inhibitor [3, 4]. Both drugs have moderate efficacy, but $20-30 \%$ of patients discontinue treatment or have dose-limiting side-effects. Thus, safe, effective treatments for IPF are urgently required. Numerous early-phase clinical trials have been performed in IPF, but few of these have progressed to phase $2 \mathrm{~b}$ or 3 trials. One of the reasons for this attrition from early- to late-phase trials is the lack of confidence that the study drug engages with its target in the human disease state. This issue is not unique to IPF.

Galectin (Gal)-3 is a central regulator of fibrosis in the lung with expression upregulated in bronchoalveolar lavage (BAL) fluid and serum of IPF patients, and further elevation observed during exacerbations $[5,6]$. The profibrotic function of Gal-3 is multifactorial due to its ability to cross-link and promote signalling via multiple cell surface receptors including integrins and growth factor receptors [7], such as transforming growth factor- $\beta[6,8]$, vascular endothelial growth factor [9] and platelet-derived growth factor (PDGF) receptors $[10,11]$. Constitutive global deficiency of Gal-3 leads to attenuated fibrosis in murine models $[6,12-14]$

TD139 is a 3,3'-bis-(4-aryltriazol-1-yl) thio-digalactoside Gal-3 inhibitor with high affinity for the Gal-3 carbohydrate recognition domain that has shown efficacy in murine models of lung fibrosis $[6,15]$. The antifibrotic potential of TD139 centres around the inhibition of the recruitment and expansion of Gal-3-secreting macrophages that drive local myofibroblast activation [14, 16, 17]. TD139 has been shown pre-clinically to exhibit effects on all of the key IPF cell types: modulating macrophage phenotype/Gal-3 expression and fibroblast activation, reducing the effects of key profibrotic growth factors that act on myofibroblasts, and inhibiting epithelial-mesenchymal transition $[6,15,16,18,19]$.

The aim of this study was to investigate the safety, pharmacokinetics (PK) and pharmacodynamics (PD) profile of TD139 when administered via a dry powder inhaler in healthy subjects and IPF patients.

\section{Materials and methods}

\section{Study design}

This study was a randomised, double-blind, multicentre, placebo-controlled, dose-escalation study investigating the safety, tolerability, PK and PD properties of TD139, a Gal-3 inhibitor administered by inhalation, in healthy subjects (part 1) and patients with IPF (part 2). The study is registered at ClinicalTrials.gov with identifier number NCT02257177. Part 1 was completed at Simbec Research (Merthyr Tydfil, UK) and part 2 was undertaken at four hospitals in the UK (Edinburgh Royal Infirmary, Edinburgh; Royal Brompton Hospital, London; Royal Victoria Infirmary, Newcastle upon Tyne; and the Royal Devon and Exeter NHS Trust, Exeter). Independent ethics committee approval was obtained prior to initiation of the study. Screening in study centres was performed within 28 days prior to randomisation and first dosing (day 1). The trial was registered prospectively as a two-part phase 1 study: part 1 in healthy volunteers commenced in September 2014 and part 2 in patients with IPF commenced in March 2015.

In part 1 of the study, following an initial screening process, subjects were randomly assigned in a $4: 2$ ratio to receive a single dose of $\operatorname{TD} 139(0.15,1.5,3,10,20$ or $50 \mathrm{mg})$ or placebo via a dry powder inhaler (Plastiape/Berry Bramlage, Lohne, Germany) (randomisation code generated using the PROC PLAN procedure of SAS). All subjects, site and study sponsor personnel were blinded to the study group assignments throughout the study. TD139 or placebo was administered in the morning of day 1 fasted

This article has an editorial commentary: https://doi.org/10.1183/13993003.00691-2021

This article has supplementary material available from erj.ersjournals.com

This study is registered at ClinicalTrials.gov with identifier number NCT02257177. Anonymised patient-level data will be made available to independent researchers, subject to review by an independent panel, at www. clinicalstudydatarequest.com within 6 months of publication. To protect the privacy of patients and individuals involved in their studies, the trial sponsor (Galecto) does not publicly disclose patient-level data.

Received: 29 June 2020 | Accepted: 5 Nov 2020 
(after an overnight fast of at least $10 \mathrm{~h}$ ). Subjects were discharged $24 \mathrm{~h}$ post-dose (day 2) provided there were no safety concerns, and returned for three outpatient visits on days 3, 8 and 14 post-dose, with a post-study follow-up between days 26 and 30. Safety and PK assessments were made at predetermined time-points.

In part 2 of the study, following an initial screening process, patients with a multidisciplinary team (MDT)-confirmed diagnosis of definite or probable IPF according to current international consensus criteria [1] were randomly assigned in a 5:3 ratio to receive a dose of TD139 (0.3, 3 or $10 \mathrm{mg})$ or placebo via a dry powder inhaler (Plastiape/Berry Bramlage) once daily for 14 days (randomisation code generated using the PROC PLAN procedure of SAS). All patients, investigators, sites and study sponsor personnel were blinded to the study group assignments throughout the study. Patients underwent study-specific assessments on days 2, 3, 7, 14 and 15, and a post-study assessment was carried out 26-30 days after the last day of the study period. Assessments included vital signs (supine blood pressure, pulse, oxygen saturation, oral temperature and respiratory rate), spirometry, 12-lead ECG, physical examination, laboratory safety screen (haematology, biochemistry, urinalysis and urinary drugs of abuse screen) and blood sampling for PK measurements. All adverse events and concomitant medications were recorded from the time of the screening visit until the post-study follow-up visit. BAL was performed at day -1 ( $24 \mathrm{~h}$ before the first dose) and at day 14 ( $2 \mathrm{~h}$ after the last dose). The BAL procedure was performed as per international guidelines [20]. Briefly, $240 \mathrm{~mL}$ of $0.9 \%$ saline at room temperature was instilled in $40 \mathrm{~mL}$ aliquots into the right middle lobe and aspirated by suction after each aliquot.

Following completion of dosing for the final subject in each dosing cohort, an interim dose review meeting was held with a safety committee consisting of the principal investigator, medical monitor, pharmacokineticist and statistician to review the safety, tolerability and PK data (study design schematic shown in figure 1). The study statistician and pharmacokineticist were unblinded at the end of each cohort in order to conduct the analyses required for dose review decisions. The primary end-point was the safety and tolerability of single or multiple doses of TD139 in healthy subjects or IPF patients, respectively. Secondary end-points included evaluation of the PK properties of TD139, expression of Gal-3 in lung and blood, and changes in lung macrophage phenotype and plasma biomarkers following treatment.

\section{Study participants}

The healthy subject population was comprised of 36 male subjects aged 23-53 years. The patient population was comprised of 24 patients aged 45-85 years with a consensus MDT diagnosis of IPF based on the American Thoracic Society/European Respiratory Society/Japanese Respiratory Society/Latin American Thoracic Association criteria [1]. Subjects had forced vital capacity (FVC) $\geqslant 45 \%$ predicted, forced expiratory volume in $1 \mathrm{~s}\left(\mathrm{FEV}_{1}\right) / \mathrm{FVC} \geqslant 0.7$, oxygen saturation $>90 \%$ on air, diffusing capacity of the lung for carbon monoxide $>25 \%$ predicted, and were judged by investigators to be safely able to undergo BAL prior to drug treatment and again after 14 days of treatment. Patients receiving oral corticosteroids or any approved or investigational antifibrotic therapies for IPF within 4 weeks of initial screening were excluded from the study. The baseline demographics are shown in tables 1 and 2, and a full list of inclusion and exclusion criteria is presented in supplementary table S1.

\section{Study drug}

TD139 was administered once daily via a dry powder inhaler. For part 2, a dose range between 0.3 and $10 \mathrm{mg}$ once daily was predicted to provide pharmacologically relevant exposures based on in vitro and in vivo pharmacology studies.

\section{Pharmacokinetics}

Full plasma PK profiles were conducted on days 1 (first day of dosing) and 14 (last day of dosing). Plasma, BAL alveolar macrophage and BAL fluid concentrations of TD139 were analysed using a validated analytical method based on protein precipitation, followed by high-performance liquid chromatography/ mass spectrometry analysis. Standard noncompartmental methods were applied to derive PK parameters using Phoenix WinNonlin version 6.3 (Certara, Sheffield, UK). Reported parameters were maximum plasma concentration $\left(C_{\max }\right)$, minimal plasma concentration $\left(24 \mathrm{~h}\right.$ post-dose) $\left(C_{\min }\right)$, plasma half-life $\left(T_{1 / 2}\right)$ and area under the concentration time curve from time of dosing extrapolated to infinity $\left(\mathrm{AUC}_{0-\mathrm{inf}}\right)$. Drug compartment measurements in BAL alveolar macrophages and fluid were determined as described previously [21]. Epithelial lining fluid (ELF) concentrations of TD139 were calculated as described previously with urea measured using a QuantiChrom urea assay kit (BioAssay Systems, CA, USA) [22].

\section{Pharmacodynamics end-points}

BAL cells were separated from fluid by centrifugation, and cells were collected, stained with antibodies (supplementary material) and then fixed before analysis using a LSR Fortessa flow cytometer (Becton 
a)

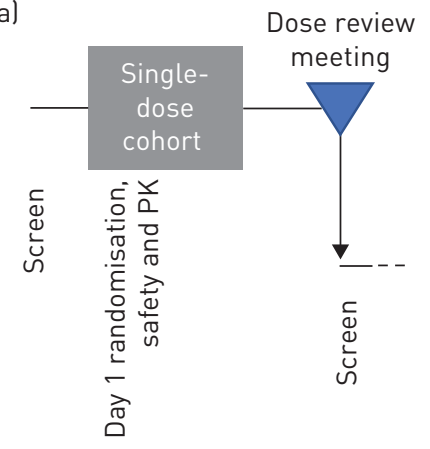

b)

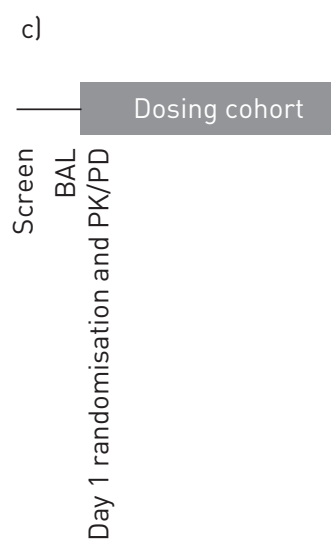

Dose review meeting

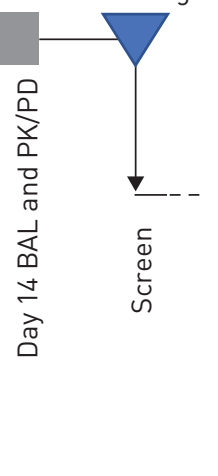

d)
Assessed for eligibility (n=71)
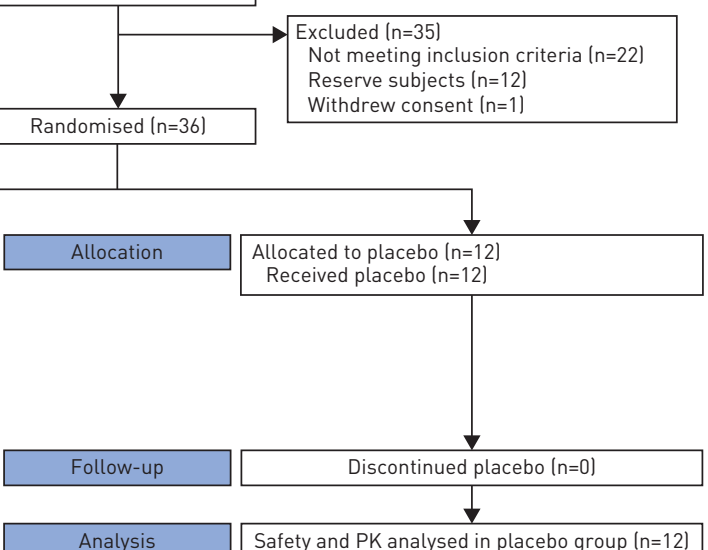

FIGURE 1 Schematic of the study design and CONSORT (Consolidated Standards of Reporting Trials) diagrams. PK: pharmacokinetics; BAL: bronchoalveolar lavage; PD: pharmacodynamics. a) Part 1 (healthy subjects). Each single-dose cohort consisted of six healthy subjects randomised 4:2 to active and placebo. A minimum of five patients' data was required in each cohort prior to data review. b) CONSORT diagram for part 1. c) Part 2 (idiopathic pulmonary fibrosis patients). Each repeat-dose cohort consisted of eight subjects randomised 5:3 to active and placebo. A minimum of seven patients' data was required in each cohort prior to data review. d) CONSORT diagram for part 2.

Dickinson, Franklin Lakes, NJ, USA). Data were analysed using FlowJo software (Tree Star, Ashland, OR, USA). Macrophages were identified as having high side scatter properties and human leukocyte antigen-DR positivity. Gal-3 expression (mean fluorescence intensity) was determined from the macrophage gate (supplementary figures S1 and S2). A range of pre-selected plasma biomarkers known to be involved in Gal-3 pathways and/or suggested to be putative biomarkers in IPF were also investigated (supplementary material and supplementary table S2). Samples were analysed by magnetic Luminex assay (R\&D Systems, Minneapolis, MN, USA) or ELISA.

TABLE 1 Baseline demographics: part 1 (healthy subjects)

\begin{tabular}{|c|c|c|c|c|c|c|c|c|}
\hline & \multicolumn{6}{|c|}{ TD139 } & Placebo & Total \\
\hline Subjects & 4 & 4 & 4 & 4 & 4 & 4 & 12 & 36 \\
\hline Age years & $42.3 \pm 9.0$ & $29.8 \pm 6.8$ & $39.3 \pm 10.2$ & $29.8 \pm 2.1$ & $30.8 \pm 9.5$ & $32.8 \pm 9.9$ & $35.6 \pm 7.5$ & $34.6 \pm 8.4$ \\
\hline BMI $\mathrm{kg} \cdot \mathrm{m}^{-2}$ & $25.6 \pm 5.1$ & $30.3 \pm 2.7$ & $25.9 \pm 3.5$ & $25.1 \pm 2.7$ & $23.8 \pm 1.2$ & $25.7 \pm 1.4$ & $25.6 \pm 2.4$ & $25.9 \pm 3.1$ \\
\hline Height m & $1.84 \pm 0.12$ & $1.75 \pm 0.10$ & $1.77 \pm 0.07$ & $1.75 \pm 0.03$ & $1.73 \pm 0.05$ & $1.85 \pm 0.11$ & $1.79 \pm 0.07$ & $1.78 \pm 0.08$ \\
\hline Weight kg & $86.0 \pm 15.5$ & $93.1 \pm 12.8$ & $80.8 \pm 9.2$ & $76.0 \pm 5.9$ & $71.4 \pm 1.5$ & $88.2 \pm 13.4$ & $81.8 \pm 8.8$ & $82.3 \pm 11.0$ \\
\hline
\end{tabular}

Data are presented as $\mathrm{n}$ or mean $\pm \mathrm{SD}$. BMI: body mass index. All subjects in part 1 were male. 


\begin{tabular}{|c|c|c|c|c|c|}
\hline & \multicolumn{3}{|c|}{ TD139 } & \multirow[t]{2}{*}{ Placebo } & \multirow[t]{2}{*}{ Total } \\
\hline & $0.3 \mathrm{mg}$ & $3 \mathrm{mg}$ & $10 \mathrm{mg}$ & & \\
\hline Subjects & 5 & 5 & 5 & 9 & 24 \\
\hline Age years & $69.0 \pm 6.3$ & $73.6 \pm 5.9$ & $79.2 \pm 2.8$ & $72.9 \pm 4.6$ & $73.5 \pm 5.8$ \\
\hline \multicolumn{6}{|l|}{ Sex } \\
\hline Male & $4(80.0)$ & 5 (100.0) & 5 (100.0) & $9(100.0)$ & 23 (95.8) \\
\hline Female & 1 (20.0) & $0(0)$ & $0(0)$ & $0(0)$ & $1(4.2)$ \\
\hline$B M I \mathrm{~kg} \cdot \mathrm{m}^{-2}$ & $28.8 \pm 1.7$ & $25.0 \pm 1.4$ & $26.3 \pm 2.2$ & $28.4 \pm 2.4$ & $27.3 \pm 2.4$ \\
\hline Height $m$ & $1.73 \pm 0.06$ & $1.77 \pm 0.05$ & $1.74 \pm 0.09$ & $1.78 \pm 0.05$ & $1.76 \pm 0.06$ \\
\hline Weight kg & $86.3 \pm 3.9$ & $78.5 \pm 5.7$ & $80.1 \pm 10.6$ & $90.2 \pm 7.4$ & $84.8 \pm 8.5$ \\
\hline FVC \% pred & $108.0 \pm 19.6$ & $81.8 \pm 16.0$ & $98.0 \pm 13.1$ & $91.7 \pm 14.0$ & $94.3 \pm 16.8$ \\
\hline FEV $_{1} \%$ pred & $104.0 \pm 19.6$ & $88.4 \pm 16.8$ & $106.4 \pm 17.7$ & $94.9 \pm 13.6$ & $97.9 \pm 16.8$ \\
\hline
\end{tabular}

Data are presented as $\mathrm{n}$, mean \pm SD or $\mathrm{n}(\%)$. BMI: body mass index; FVC: forced vital capacity; $\mathrm{FEV}_{1}$ : forced expiratory volume in $1 \mathrm{~s}$.

\section{Statistical analysis}

For part 2 of the study, placebos were combined from the three dose-specific cohorts into a pooled control group ( $\mathrm{n}=9)$. For alveolar macrophage Gal-3 expression and the plasma biomarkers, ANCOVA was performed for percentage of baseline ((day 14/day 1) $\times 100)$. The ANCOVA model included effects for treatment group and baseline (day 1) value. The least square means for each treatment group, the difference in least square means for each active dose compared with control with associated 95\% two-sided confidence interval as well as the p-value for treatment difference were obtained. As some imbalance was seen in demographic and baseline characteristics between the treatment groups, a further ANCOVA model was fitted including effects for age, weight and $\mathrm{FEV}_{1}$ at baseline. These covariates were not found significant in any model and did not change the interpretation of the results, and so were removed from the final models. A Bayesian approach was taken to use these results to quantify the evidence for a positive effect of the drug for each biomarker. Under the assumption of a noninformative prior for the treatment difference, the posterior probability of a treatment effect greater than zero (percentage of baseline $<100 \%$ ) is equivalent to 1 -the one-sided lower p-value $\left(\mathrm{H}_{1}: \mu_{\text {Drug }}<\mu_{\text {Placebo }}\right)$ from this ANCOVA model. An additional analysis including dose as a continuous covariate was also performed. The Pearson correlation coefficient (r) and associated p-value were calculated for percentage of baseline and day 14 values between plasma biomarkers and alveolar macrophage Gal-3 expression. If appropriate, Fisher's exact test was performed for whether a subject had a percentage of baseline $<100 \%$ for the plasma biomarker and alveolar macrophage Gal-3 expression (yes/no) versus treatment (placebo versus $10 \mathrm{mg}$ ). The analysis is exploratory and there was no pre-planned adjustment for multiple testing; the study was not powered for this. Alveolar macrophage Gal-3 expression was considered the primary exploratory analysis with the pre-selected biomarkers secondary. A Bonferroni correction for multiple testing of the secondary end-points comparing the $10 \mathrm{mg}$ group with placebo gives a critical value of 0.0063 . Data are presented as the difference in least square means with $95 \%$ confidence interval and p-value unless otherwise stated. All analyses were performed using SAS version 9.4 (SAS Institute, Cary, NC, USA). A concentration-response model was fitted to the alveolar macrophage concentration of TD139 for each subject and the alveolar macrophage surface Gal-3 level (percentage of baseline) using Prism version 8.0 (GraphPad, San Diego, CA, USA) to generate maximum effect $\left(E_{\max }\right)$ and median inhibitory concentration $\left(\mathrm{IC}_{50}\right)$ values.

\section{Results}

The enrolment and flow of healthy subjects and IPF patients through part 1 and part 2 of the study is detailed in the CONSORT (Consolidated Standards of Reporting Trials) diagrams (figure 1). Subject disposition and demographics are presented in tables 1 and 2. A small imbalance was observed in age, weight, body mass index and $\mathrm{FEV}_{1}$; however, this was found not to impact the analysis results.

Overall, TD139 was considered safe and well tolerated at single doses up to $50 \mathrm{mg}$ and multiple inhaled doses up to $10 \mathrm{mg}$. In part 1, 28 mild treatment-emergent adverse events (TEAEs) were reported by 15 (41.7\%) subjects during the study (table 3 ). The most commonly occurring TEAE associated with TD139 was mild dysgeusia (distortion of sense of taste) (36.1\%), but with no serious tolerability issues. Cough was reported, although the incidence was low (11.1\%). There were no other TEAEs of note and in general there were no dose-related trends observed. In addition, there were no clinically significant safety findings or treatment- or dose-related changes observed in biochemistry, haematology, vital signs or 12-lead ECG 
TABLE 3 Summary of treatment-emergent adverse events (TEAEs): part 1 (healthy subjects)

\begin{tabular}{|c|c|c|c|c|c|c|c|c|}
\hline & \multicolumn{6}{|c|}{ TD139 } & Placebo & Total \\
\hline TEAEs & 0 & 0 & 2 & 7 & 7 & 10 & 2 & 28 \\
\hline \multicolumn{9}{|l|}{ Subjects reporting $\geqslant 1$ TEAE } \\
\hline TEAE & 0 & 0 & 2 (50.0) & $3(75.0)$ & $4(100.0)$ & $4(100.0)$ & $2(16.7)$ & $15(41.7)$ \\
\hline Serious TEAE & 0 & 0 & 0 & 0 & 0 & 0 & 0 & 0 \\
\hline TEAE leading to withdrawal & 0 & 0 & 0 & 0 & 0 & 0 & 0 & 0 \\
\hline \multicolumn{9}{|c|}{ Subjects with TEAE by relationship to study IMP } \\
\hline Almost definite & 0 & 0 & $1(25.0)$ & $3(75.0)$ & $4(100.0)$ & $4(100.0)$ & $2(16.7)$ & $14(38.9)$ \\
\hline Probable & 0 & 0 & 0 & 0 & $1(25.0)$ & 0 & 0 & $1(2.8)$ \\
\hline Possible & 0 & 0 & 0 & $2(50.0)$ & $1(25.0)$ & $3(75.0)$ & 0 & $6(16.7)$ \\
\hline Unlikely & 0 & 0 & $1(25.0)$ & 0 & 0 & 0 & 0 & $1(2.8)$ \\
\hline
\end{tabular}

Data are presented as $\mathrm{n}$ or $\mathrm{n}(\%)$. A subject with multiple occurrences of an adverse event is counted only once within each level of severity or relationship. IMP: investigational medicinal product.

data. In part 2, there were no treatment- or dose-related trends observed in any TEAE profile, biochemistry, haematology, vital signs or 12-lead ECG data following treatment with TD139. 20 patients had at least one TEAE (table 4); however, there were no withdrawals as a result. Most TEAEs were unrelated to the study drug and only two patients had a TEAE (diarrhoea and dysgeusia/oropharyngeal pain) considered possibly related to the study drug. There was one post-treatment severe TEAE of acute exacerbation of IPF (AE-IPF) triggered by pneumonia reported in the $10 \mathrm{mg}$ TD139 IPF group. This patient received all 14 doses of the study drug with the treatment course recorded as uneventful and pneumonia diagnosed 4 days after the last dose of TD139 following hospital admission. The pneumonia worsened, leading to death 32 days later. Infection was regarded as possibly related to the BAL procedure but unrelated to the study drug by the study investigators.

Plasma concentrations of TD139 in healthy subjects and IPF patients are presented in figures $2 \mathrm{a}$ and b, respectively, and derived PK parameters are shown in tables 5 and 6. TD139 absorption was nonlinear, increasing with dose and between day 1 and day 14. The PK profile of TD139 in IPF patients in this study was comparable to healthy volunteers; however, the exposure at the highest $10 \mathrm{mg}$ dose was much greater

TABLE 4 Summary of treatment-emergent adverse events (TEAEs): part 2 (idiopathic pulmonary fibrosis patients)

\begin{tabular}{|c|c|c|c|c|c|}
\hline & \multicolumn{3}{|c|}{ TD139 } & \multirow[t]{2}{*}{ Placebo } & \multirow[t]{2}{*}{ Total } \\
\hline & $0.3 \mathrm{mg}$ & $3 \mathrm{mg}$ & $10 \mathrm{mg}$ & & \\
\hline TEAEs & 9 & 10 & 13 & 23 & 55 \\
\hline \multicolumn{6}{|l|}{ Subjects reporting $\geqslant 1$ TEAE } \\
\hline TEAE & $4(80.0)$ & $5(100.0)$ & $4(80.0)$ & $7(77.8)$ & 20 (83.3) \\
\hline Serious TEAE & 0 & 0 & $1(20.0)$ & 0 & $1(4.2)$ \\
\hline TEAE leading to withdrawal & 0 & 0 & 0 & 0 & 0 \\
\hline \multicolumn{6}{|l|}{ Subjects with TEAE by severity } \\
\hline Mild & $2(40.0)$ & $4(80.0)$ & $1(20.0)$ & $4(44.4)$ & $11(45.8)$ \\
\hline Moderate & $2(40.0)$ & $1(20.0)$ & $2(40.0)$ & 3 (33.3) & 8 (33.3) \\
\hline Severe & 0 & 0 & $1(20.0)$ & 0 & $1(4.2)$ \\
\hline \multicolumn{6}{|c|}{ Subjects with TEAE by relationship to study IMP } \\
\hline Almost definite & 0 & 0 & 0 & 0 & 0 \\
\hline Probable & 0 & 0 & 0 & 0 & 0 \\
\hline Possible & $1(20.0)$ & $1(20.0)$ & 0 & 0 & $2(8.3)$ \\
\hline Unlikely & $1(20.0)$ & $1(20.0)$ & $2(40.0)$ & $2(22.0)$ & $6(25.0)$ \\
\hline Unrelated & $2(40.0)$ & $3(60.0)$ & $2(40.0)$ & $5(55.6)$ & $12(50.0)$ \\
\hline
\end{tabular}

Data are presented as $\mathrm{n}$ or $\mathrm{n}(\%)$. A subject with multiple occurrences of an adverse event is counted only once within each level of severity or relationship. IMP: investigational medicinal product. 

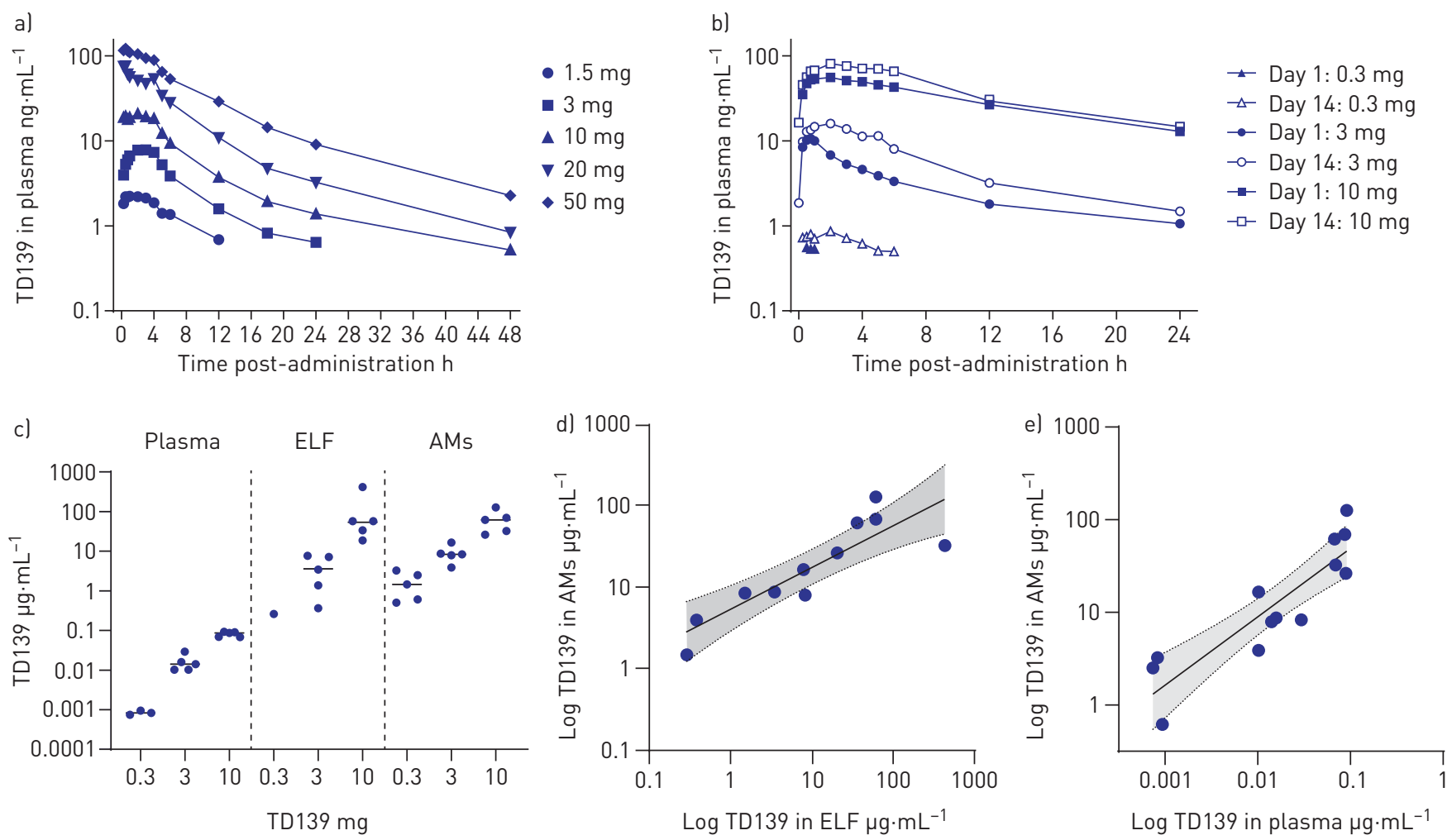

FIGURE 2 TD139 pharmacokinetics in healthy subjects and idiopathic pulmonary fibrosis (IPF) patients. ELF: epithelial lining fluid; AM: alveolar macrophage. a) Healthy subjects: log-linear mean plasma concentration of TD139 versus time over $48 \mathrm{~h}$ following a single dose of drug. b) IPF patients: log-linear mean plasma concentration of TD139 versus time over $24 \mathrm{~h}$ following the first dose of drug on day 1 or the last dose on day 14. c) Log-linear individual measured concentrations (median) in plasma (total), ELF and AMs at $2 \mathrm{~h}$ post-administration of 0.3, 3 and $10 \mathrm{mg}$ TD139 on day 14. d) Correlation between concentrations of TD139 in ELF and AMs for all active cohorts on day $14(\mathrm{r}=0.87(95 \% \mathrm{Cl} 0.56-0.96) ; \mathrm{p}<0.001)$. e) Correlation between concentrations of TD139 in plasma (total) and AMs for all active cohorts on day 14 ( $r=0.89$ (95\% Cl 0.65-0.96); $p<0.0001)$. Shading represents $95 \% \mathrm{Cl}$ of the linear fit. For reference, the lower limit of detection in plasma was $0.5 \mathrm{ng} \cdot \mathrm{mL}^{-1}$ and in BAL fluid was $5 \mathrm{ng} \cdot \mathrm{mL}^{-1}$.

in patients with IPF compared with the same dose in healthy subjects. Thus, a 3.3 -fold increase in dose to $10 \mathrm{mg}$ in patients with IPF resulted in a disproportionately high increase in exposure on day 1, with a

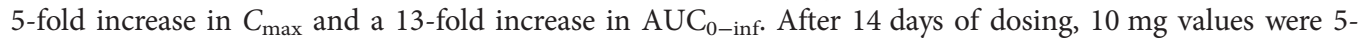
and 8 -fold greater, respectively, with $\sim 50 \%$ accumulation. With a $T_{1 / 2}$ of $\sim 8 \mathrm{~h}$ and a dosing interval of $24 \mathrm{~h}$, the expected accumulation would be $\sim 14 \%$. These data suggest that TD139 is retained in the lungs but to a lesser extent in IPF patients compared with healthy subjects and may be saturable, with a slightly greater accumulation in the plasma than expected over time and a much higher systemic exposure with increasing dose. TD139 concentration was assayed in alveolar macrophages derived from patients' day 14 BAL, in ELF and in plasma (figure 2c). There was a dose-dependent correlation between TD139

TABLE 5 Summary of plasma-derived pharmacokinetics parameters: part 1 (healthy subjects)

TD139

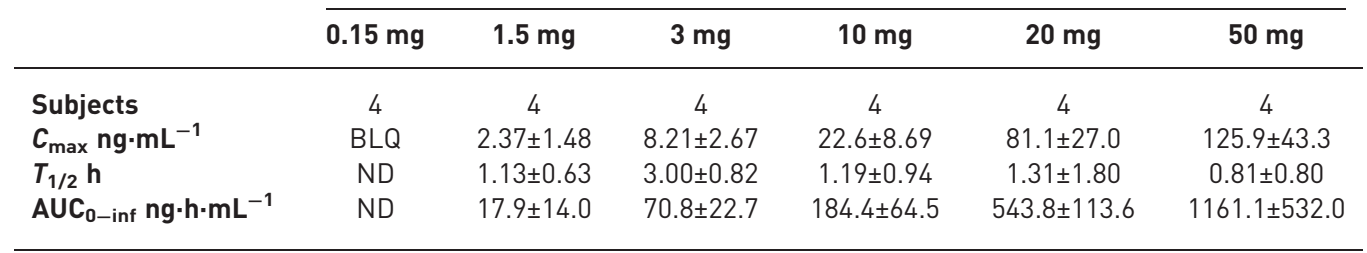

Data are presented as $\mathrm{n}$ or mean $\pm \mathrm{SD}$. $C_{\text {max }}$ : maximum plasma concentration; $T_{1 / 2}$ : plasma half-life; $\mathrm{AUC}_{0 \text {-inf: }}$ area under the curve extrapolated to infinity from dosing time, based on the last observed concentration at $24 \mathrm{~h}$ post-dose; BLQ: below limit of quantification $\left(0.5 \mathrm{ng} \cdot \mathrm{mL}^{-1}\right)$; ND: not determined due to insufficient data. 
TABLE 6 Summary of plasma-derived pharmacokinetics parameters: part 2 lidiopathic pulmonary fibrosis patients)

\begin{tabular}{|c|c|c|c|}
\hline & \multicolumn{3}{|c|}{ TD139 } \\
\hline & $0.3 \mathrm{mg}$ & $3 \mathrm{mg}$ & $10 \mathrm{mg}$ \\
\hline Subjects & 5 & 5 & 5 \\
\hline \multicolumn{4}{|c|}{$C_{\max } \mathrm{ng} \cdot \mathrm{mL}^{-1}$} \\
\hline Day 1 & $0.55 \pm 0.02$ & $10.6 \pm 11.1$ & $56.3 \pm 19.3$ \\
\hline Day 14 & $0.86 \pm 0.25$ & $16.5 \pm 8.34$ & $82.8 \pm 11.8$ \\
\hline \multicolumn{4}{|c|}{$c_{\min } \mathrm{ng} \cdot \mathrm{mL}^{-1}$} \\
\hline Day 1 & $B L Q$ & $1.06 \pm 0.43$ & $12.9 \pm 7.88$ \\
\hline Day 14 & $B L Q$ & $1.48 \pm 0.51$ & $14.7 \pm 8.64$ \\
\hline \multicolumn{4}{|l|}{$T_{1 / 2} \mathrm{~h}$} \\
\hline Day 1 & ND & $6.36 \pm 2.09$ & $9.69 \pm 1.70$ \\
\hline Day 14 & ND & $6.91 \pm 1.40$ & $8.20 \pm 2.26$ \\
\hline \multicolumn{4}{|c|}{$A U C_{0-\text { inf }} \mathrm{ng} \cdot \mathrm{h} \cdot \mathrm{mL}^{-1}$} \\
\hline Day 1 & ND & $69 \pm 50$ & $921 \pm 479$ \\
\hline Day 14 & ND & $149 \pm 47$ & $1150 \pm 462$ \\
\hline
\end{tabular}

concentrations measured between the three compartments (figure $2 \mathrm{~d}$ and e). The concentration of TD139 in BAL macrophages was between 567- and 1930-fold higher than from systemic exposure at $2 \mathrm{~h}$ post-dosing on day 14 .

Gal-3 expression on BAL macrophages was reduced by administration of TD139 (figure 3a and supplementary figure S3). The percentage of baseline at day 14 differed between the $3 \mathrm{mg}(-38.66 \%(95 \%$ CI $-69.59--7.73 \%) ; \mathrm{p}=0.017)$ and $10 \mathrm{mg}(-44.63 \%$ (95\% CI $-80.44--8.81 \%) ; \mathrm{p}=0.0173)$ dose groups compared with placebo. A concentration-dependent reduction in cell surface Gal-3 was observed in BAL macrophages, with a near-maximal effect observed in the $10 \mathrm{mg}$ TD139 cohort (figure $3 \mathrm{~b}$ and $\mathrm{c}$ ). The concentrations required to induce this effect were several orders of magnitude greater than that present in the systemic circulation (figure $3 b$ ).

Circulating Gal-3 plasma concentrations (percentage of baseline) were reduced in the highest dose group (10 mg) versus placebo (-67.63\% (95\% CI -126.86- -8.40\%); $\mathrm{p}=0.0275)$ and correlated with the change in expression of Gal-3 on BAL macrophages at day 14 (figure 4). Fisher's exact test of whether a subject has percentage of baseline $<100 \%$ for Gal-3 plasma and Gal-3 on BAL macrophages compared for treatment groups found a difference between the $10 \mathrm{mg}$ versus placebo groups $(\mathrm{p}=0.031)$.

Plasma from patients at day -1 and day 14 was analysed for the expression of a panel of pre-specified inflammatory and fibrosis biomarkers. A disease relevance score was retrospectively assigned to these biomarkers to rank them for importance in IPF progression (table 7 and supplementary table S2). When comparing placebo with the $10 \mathrm{mg}$ TD139 group after adjusting for baseline values, five of the 11 high-relevance plasma markers (PDGF-BB, plasminogen activator inhibitor (PAI)-1, Gal-3, CCL18 and YKL-40) with a link to IPF pathogenesis were reduced $(\mathrm{p}<0.05)$. The Bayesian probability that the treatment effect is greater than zero (percentage of baseline $<100 \%$ ) in the $10 \mathrm{mg}$ group is $>98 \%$ (figure 4 , table 7 and supplementary table S2). Another four biomarkers with medium or high relevance ranking (matrix metalloproteinase (MMP)-8, PDGF-AA, hepatocyte growth factor and MMP-1) were impacted by treatment with $>90 \%$ probability (table 7 ). Similar results were found when dose was included as a continuous covariate (supplementary table S4). There was also a good correlation between the change in lung Gal-3 concentration and the change in plasma levels of the high-relevance biomarkers in the $10 \mathrm{mg}$ group (figure 4). Overall, the differential impact of TD139 was predominantly on profibrotic versus pro-inflammatory markers. In addition, there was no significant change in any biomarker between day -1 and day 14 in the placebo group (table 7).

\section{Discussion}

This is the first study to evaluate the pharmacology of a novel therapeutic targeting Gal-3 inhibition in IPF. In addition, it is also the first inhaled therapeutic to be investigated in an IPF clinical study. A large 

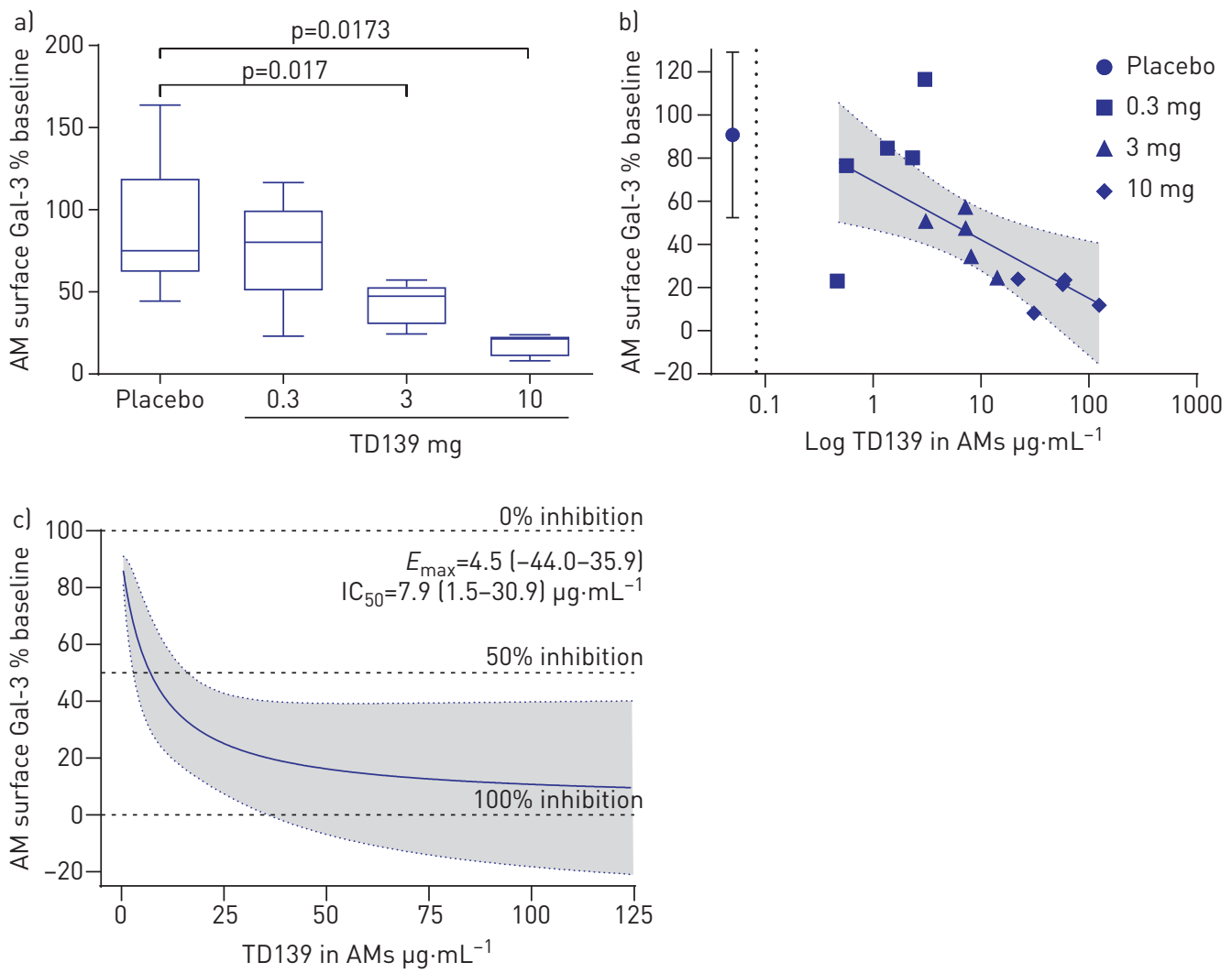

FIGURE 3 Galectin (Gal)-3 changes in alveolar macrophages (AMs). a) Box plot of TD139 dose-dependent effect on percentage change from baseline in surface macrophage Gal-3 levels. Boxes indicate median and interquartile range; whiskers indicate minimum-maximum range. p-value from ANCOVA model adjusted for baseline. b) Concentration-dependent effect between TD139 in AMs and percentage of baseline in surface macrophage Gal-3, with maximum plasma concentration $\left(C_{\max }\right)$ at $10 \mathrm{mg}$ dose (dotted line) shown for comparison ( $r=-0.63(95 \% \mathrm{Cl}-0.86-0.18) ; \mathrm{p}=0.011)$. Shading represents $95 \% \mathrm{Cl}$ of the linear fit. Individual subject data are labelled by dose. c) Fitted maximum effect model of surface macrophage Gal-3 (percentage of baseline) versus macrophage TD139 concentration. Maximum effect ( $E_{\max }$, AM surface Gal-3 percentage of baseline) and median inhibitory concentration $\left(\mathrm{IC}_{50}\right)$ values are shown. Shading represents $95 \% \mathrm{Cl}$ of the fitted maximum effect model.

body of evidence points to Gal-3 being an important mediator of fibrosis across multiple organs and pre-clinical work with TD139 has demonstrated the antifibrotic potential of targeting Gal-3 in lung fibrosis $[6,15]$. An initial study in healthy subjects (part 1) demonstrated that single doses of TD139 between 0.15 and $50 \mathrm{mg}$ were well tolerated. Reported adverse events were all judged to be mild and inhaled administration of TD139 in this healthy subject study demonstrated a favourable PK profile that supported undertaking a multiple, ascending dose study in individuals with IPF (part 2).

Daily inhaled administration of TD139 for 14 days via a dry powder inhaler was safe and well tolerated by patients with IPF, and the adverse events observed were mild in severity. None of the TEAEs resulted in discontinuation of treatment. The SAE in this study was deemed to be a fatal AE-IPF. The subject experienced new respiratory symptoms 2 days after the second BAL and was initially treated for presumed infection but subsequently went on to develop AE-IPF according to the then relevant 2011 criteria [1]. Molyneaux et al. [23] have recently reported on the safety of BAL in IPF in a large prospective cohort. However, we are aware that there are published data that indicate that BAL could cause AE-IPF but this is uncommon or rare [24]. Repeat BAL could plausibly increase this risk, e.g. if the first BAL induced a "priming" environment that could trigger an AE-IPF with a second BAL. A number of studies, including the current study, have successfully repeated BAL in IPF subjects with a cumulative experience of more than 80 subjects and no reports of procedure-related adverse events $[25,26]$. The interval of 14 days between BALs in this study was determined in part to mitigate against this hypothetical situation.

Inhaled administration of TD139 resulted in measurable, dose-dependent levels of the drug in plasma, ELF and alveolar macrophages. The PK profile of TD139 in individuals with IPF was consistent and 


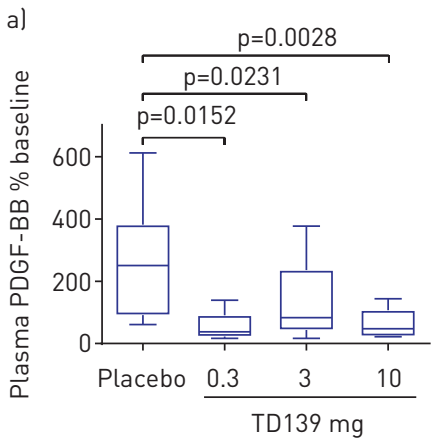

e)

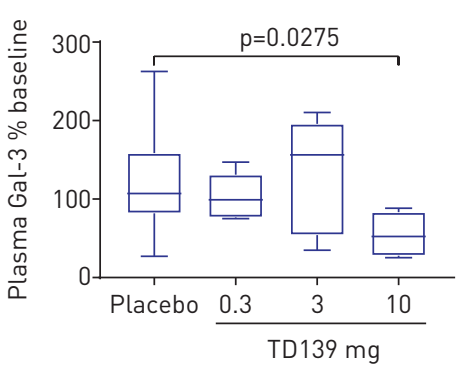

i)

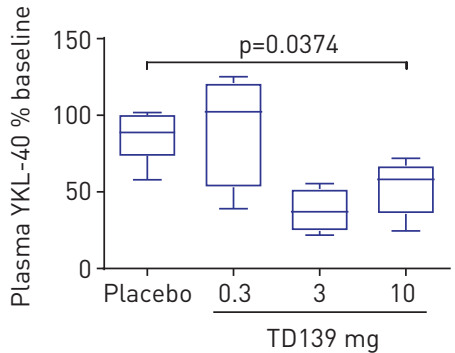

b)

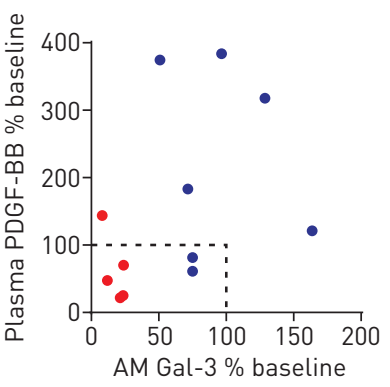

f)

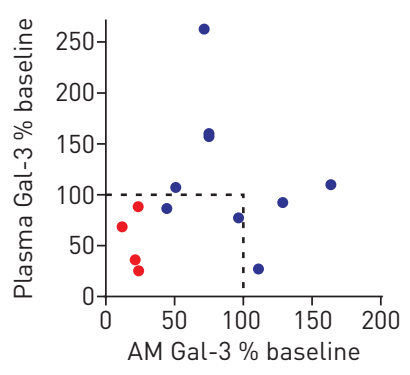

j)

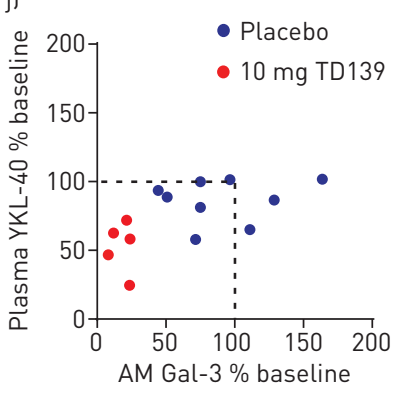

c)

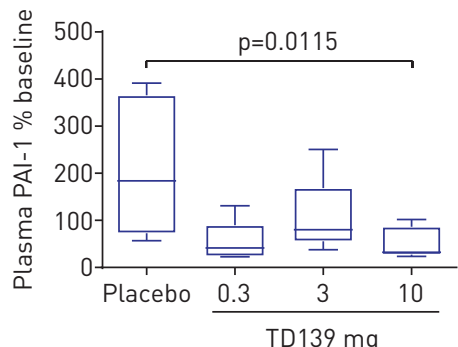

g)

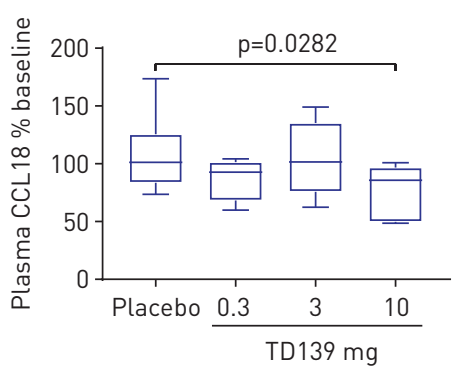

d)

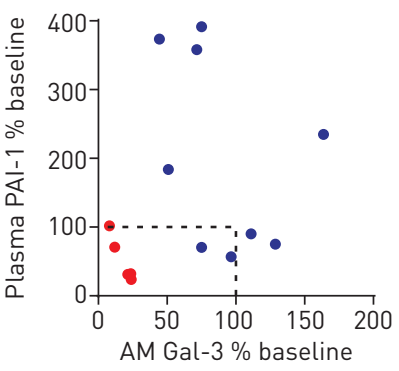

h)

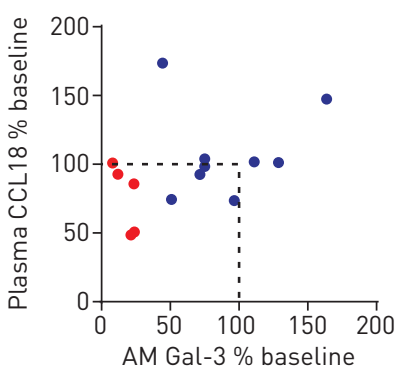

FIGURE 4 Biomarker changes in plasma. PDGF: platelet-derived growth factor; AM: alveolar macrophage; PAI: plasminogen activator inhibitor;

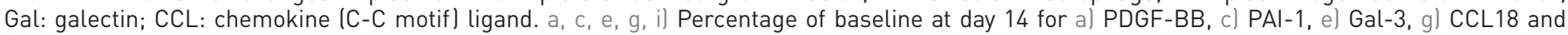
i) YKL-40 in the 0.3, 3 and $10 \mathrm{mg}$ TD139 dose groups or placebo. Boxes indicate median and interquartile range; whiskers indicate minimummaximum range. p-value from ANCOVA model adjusted for baseline. b, $d, f, h$, j) Correlation between percentage of baseline in b) PDGF-BB, d) PAl-1, f) Gal-3, h) CCL18 and j) YKL-40 versus the change in lung macrophage Gal-3 expression in the placebo and 10 mg TD139 groups. Dotted

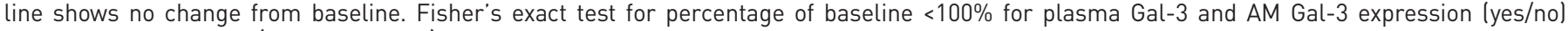
versus treatment group ( $p$ lacebo, $10 \mathrm{mg}$ ) $\mathrm{p}=0.031$.

characterised by low inter- and intra-patient variability. The PK profile in subjects with IPF was comparable to the profile in healthy subjects, although the exposure in IPF was much higher at the $10 \mathrm{mg}$

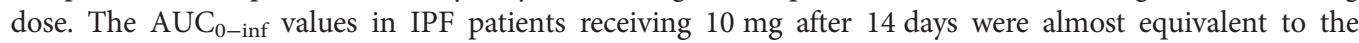
$50 \mathrm{mg}$ values in healthy subjects receiving a single dose. Loss of alveolar barrier integrity with increased epithelial permeability is well described in the fibrotic lung [27], which may account for the high systemic levels observed in IPF patients with TD139. The variability of the plasma levels at day 1 was larger than at day 14 for all three dose levels, indicating that patients may achieve a more consistent and reproducible drug exposure as they become accustomed to using the inhaler. Drug levels in the lung determined in ELF and alveolar macrophages showed a strong correlation with plasma levels, allowing a model for achieved lung exposure to be built based on the levels in the systemic circulation. This, combined with the plasma profile observed for TD139, suggested a sustained release of TD139 from the lung into the systemic compartment, demonstrating an extended lung retention. This would be predicted to achieve a prolonged inhibition of Gal-3 in the lung over a $24 \mathrm{~h}$ dosing period.

The Gal-3 levels on alveolar macrophages were inhibited by TD139 in a concentration-dependent manner, with the PK/PD relationship defined in the target compartment and the $10 \mathrm{mg}$ dose causing almost maximal inhibition. In addition, the highest dose of TD139 also decreased circulating Gal-3, which correlated with a reduction in several fibrosis-related biomarkers. Four highly relevant disease biomarkers, 
TABLE 7 Summary of statistical analysis of plasma pharmacodynamics biomarkers

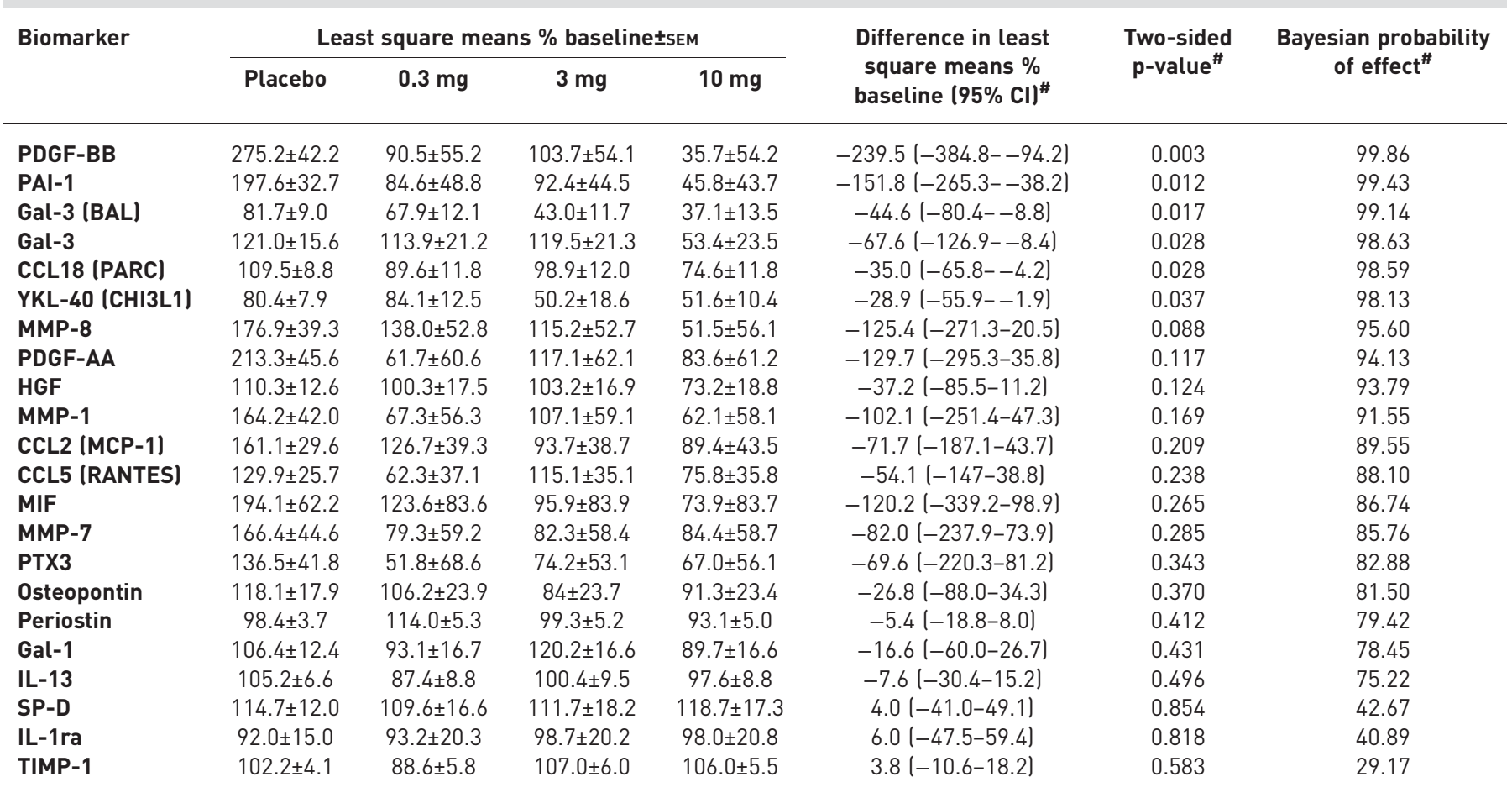

PDGF: platelet-derived growth factor; PAI: plasminogen activator inhibitor; Gal: galectin; BAL: bronchoalveolar lavage; CCL: chemokine (C-C motif) ligand; PARC: pulmonary and activation-regulated chemokine; CHI3L1: chitinase-3-like protein 1; MMP: matrix metalloproteinase; HGF: hepatocyte growth factor; MCP: monocyte chemoattractant protein; RANTES: regulated on activation, normal T-cell expressed and secreted; MIF: macrophage migration inhibitory factor; PTX: pentraxin; IL: interleukin; SP-D: surfactant protein D; ra: receptor antagonist; TIMP: tissue inhibitor of metalloproteinases; CXCL: chemokine (C-X-C motif) ligand. For the following biomarkers, the majority of values were below the lower level of quantification and therefore were not analysed: amphiregulin, CCL26 (eotaxin), CXCL1 (GRO $\alpha$ ), CXCL10 (IP-10), epidermal growth factor, IL-8, IL-10, IL-25, IL-33, interferon- $\gamma$ and tumour necrosis factor- $\alpha$. \#: results from an ANCOVA model including effects for treatment group and baseline value with the Bayesian probability of effect of the $10 \mathrm{mg}$ TD139 group versus placebo shown.

i.e. PDGF-BB [28-31], CCL18 [32, 33], PAI-1 [34-38] and YKL-40 [39-41], have been shown to have prognostic significance in IPF and have well-described effects on myofibroblast activity in vitro. These biomarkers were reduced from baseline for the $10 \mathrm{mg}$ dose group compared with placebo and offer a less invasive measure of PD effects going forward into the next phase of clinical development for TD139. The partial reduction in BAL macrophage Gal-3 in the 0.3 and $3 \mathrm{mg}$ groups was associated with a reduction in some biomarkers, e.g. PDGF-BB in plasma. Due to the pleiotropic nature of Gal-3 in fibrotic disease and the many signalling pathways it influences, it could be hypothesised that some could be more sensitive to Gal-3 modulation than others. This could result in maximal downstream inhibition of biomarkers such as PDGF-BB with only a partial inhibition of Gal-3.

In a post hoc analysis of the ASCEND and CAPACITY trials, both YKL-40 and CCL18 were prognostic for progression in the test cohort but only CCL18 was consistently prognostic for a change in FVC in both the test and replication cohorts [42]. However, of note there was no association between pirfenidone treatment and the longitudinal concentration of any biomarker [42]. In another study comparing treatment-naive IPF patients with those on antifibrotic treatment (pirfenidone or nintedanib), CA-125, CXCL13, MMP-7, YKL-40 and osteopontin predicted differential transplant-free survival in treated patients but at higher thresholds than treatment-naive individuals [43]. There is therefore substantial evidence that several biomarkers are related to disease severity and prognosis, particularly YKL-40 and CCL18, but no conclusive study that relates biomarker changes in currently approved antifibrotic therapy to survival.

The limitations of this study are the small sample size, short duration of treatment and mild severity of disease. It is therefore encouraging that TD139 has demonstrated evidence of shifts in these biomarkers despite these limitations. 
In conclusion, inhaled dosing of TD139 for 14 days to individuals with IPF has been shown to be safe and well tolerated. TD139 effectively engages with Gal-3 in the alveolar space and is associated with favourable shifts in mediators that plausibly drive lung fibrosis. These data provide the information necessary for selecting the optimal dosing strategy for TD139, and strongly support performing longer and larger trials in individuals with IPF to assess antifibrotic efficacy and the effect on lung function. A phase $2 b$ study with TD139 in individuals with IPF is now underway (GALACTIC-1; ClinicalTrials.gov: NCT03832946).

This article has been revised according to the correction published in the April 2022 issue of the European Respiratory Journal.

Author contributions: A.C. MacKinnon, N. Hirani, T.M. Maher and P. Ford designed and performed experiments, conducted data/statistical analysis and co-wrote the manuscript. R.J. Slack and L. Keeling conducted data/statistical analysis and co-wrote the manuscript. A.J. Simpson and M.A. Gibbons were clinical leads on the project, consented participants in the study, collected and analysed patient samples, and edited the manuscript. L. Paul, P.L. Molyneaux, W. Funston, I.A. Forrest and L. Nicol consented participants in the study, and collected and analysed patient samples. R. Mills, D. Humphries, U. Karmakar and F. Li collected human samples, analysed patient samples and edited the manuscript. F. Zetterberg and U.J. Nilsson designed the compound and edited the manuscript. H. Leffler, T. Sethi, L. Gravelle and S. Tantawi provided supporting data for the project, provided the compound and edited the manuscript. H. Schambye and A. Pedersen designed and supervised the study, edited the manuscript and provided financial support.

Conflict of interest: N. Hirani reports grants from Galecto Biotech, during the conduct of the study. A.C. MacKinnon reports personal fees from Galecto Biotech, outside the submitted work; and has a patent CA2,794,066 issued, a patent US13/832,672 issued and a patent WO/2014/067986 pending (all patents are fully owned by Galecto Biotech). L. Nicol reports grants from Galecto Biotech, during the conduct of the study; personal fees for lectures from Boehringer Ingelheim, outside the submitted work. P. Ford reports personal fees and nonfinancial support from Galecto, during the conduct of the study; and has a patent TD139 issued. H. Schambye reports personal fees from Galecto Inc, outside the submitted work; and has a patent WO/2016/180483 pending (fully owned by Galecto Biotech). A. Pedersen reports personal fees from Galecto Biotech, outside the submitted work. U.J. Nilsson has a patent CA2,794,066 issued, a patent US13/832,672 issued, a patent WO/2014/067986 pending, a patent $\mathrm{WO} / 2005 / 113569$ pending and a patent $\mathrm{WO} / 2009 /$ 139719 pending (all patents are fully owned by Galecto Biotech). H. Leffler has a patent CA2,794,066 issued, a patent US13/832,672 issued, a patent WO/2014/067986 pending and a patent WO/2005/113569 pending (all patents are fully owned by Galecto Biotech). T. Sethi reports personal fees from Galecto Biotech, outside the submitted work; and has a patent CA2,794,066 issued, a patent US13/832,672 issued and a patent WO/2014/067986 pending (patents are fully owned by Galecto Biotech). S. Tantawi reports personal fees from Galecto Biotech, outside the submitted work. L. Gravelle reports personal fees from Galecto Biotech, outside the submitted work; and has a patent WO/2017/103109 pending (fully owned by Galecto Biotech). R.J. Slack reports personal fees from Galecto Biotech, outside the submitted work. R. Mills has nothing to disclose. U. Karmakar has nothing to disclose. D. Humphries has nothing to disclose. F. Zetterberg reports personal fees from Galecto Biotech, outside the submitted work. L. Keeling has nothing to disclose. L. Paul has nothing to disclose. P.L. Molyneaux has, via his institution, received industry-academic funding from AstraZeneca and has received speaker and consultancy fees from Boehringer Ingelheim and Hoffman-La Roche, outside the submitted work. F. Li has nothing to disclose. W. Funston has nothing to disclose. I.A. Forrest reports personal fees for consultancy and meeting attendance from Boehringer Ingelheim, personal fees for lectures and meeting attendance from Roche Ltd, outside the submitted work. A.J. Simpson has nothing to disclose. M.A. Gibbons has nothing to disclose. T.M. Maher has, via his institution, received industry-academic funding from AstraZeneca and GlaxoSmithKline R\&D, and has received consultancy or speaker fees from AstraZeneca, Bayer, Blade Therapeutics, Boehringer Ingelheim, Bristol-Myers Squibb, Galapagos, GlaxoSmithKline R\&D, Indalo, Novartis, Pliant, Respivant, Roche and Samumed.

Support statement: This work was funded by Galecto Inc. Funding information for this article has been deposited with the Crossref Funder Registry.

\section{References}

1 Raghu G, Collard HR, Egan JJ, et al. An official ATS/ERS/JRS/ALAT statement: idiopathic pulmonary fibrosis: evidence-based guidelines for diagnosis and management. Am J Respir Crit Care Med 2011; 183: 788-824.

2 King TE Jr, Pardo A, Selman M. Idiopathic pulmonary fibrosis. Lancet 2011; 378: 1949-1961.

3 King TE Jr, Bradford WZ, Castro-Bernardini S, et al. A phase 3 trial of pirfenidone in patients with idiopathic pulmonary fibrosis. N Engl J Med 2014; 370: 2083-2092.

4 Richeldi L, du Bois RM, Raghu G, et al. Efficacy and safety of nintedanib in idiopathic pulmonary fibrosis. $N$ Engl J Med 2014; 370: 2071-2082.

5 Nishi Y, Sano H, Kawashima T, et al. Role of galectin-3 in human pulmonary fibrosis. Allergol Int 2007; 56: $57-65$.

6 Mackinnon AC, Gibbons MA, Farnworth SL, et al. Regulation of transforming growth factor- $\beta 1$-driven lung fibrosis by galectin-3. Am J Respir Crit Care Med 2012; 185: 537-546.

7 Partridge EA, Le Roy C, Di Guglielmo GM, et al. Regulation of cytokine receptors by Golgi $N$-glycan processing and endocytosis. Science 2004; 306: 120-124.

8 Shen H, Wang J, Min J, et al. Activation of TGF- $\beta 1 / \alpha-S M A / C o l$ I profibrotic pathway in fibroblasts by galectin-3 contributes to atrial fibrosis in experimental models and patients. Cell Physiol Biochem 2018; 47: 851-863.

9 Chen WS, Cao Z, Leffler H, et al. Galectin-3 inhibition by a small-molecule inhibitor reduces both pathological corneal neovascularization and fibrosis. Invest Ophthalmol Vis Sci 2017; 58: 9-20.

10 Guo SM, Feng ZM. Galectin-3 mediates the effect of PDGF on pulmonary arterial hypertension. Int J Clin Exp Med 2015; 8: 15302-15307. 
11 Jastrzebski K, Zdzalik-Bielecka D, Maminska A, et al. Multiple routes of endocytic internalization of PDGFR $\beta$ contribute to PDGF-induced STAT3 signaling. J Cell Sci 2017; 130: 577-589.

12 Henderson NC, Mackinnon AC, Farnworth SL, et al. Galectin-3 regulates myofibroblast activation and hepatic fibrosis. Proc Natl Acad Sci USA 2006; 103: 5060-5065.

13 MacKinnon AC, Liu X, Hadoke PW, et al. Inhibition of galectin-3 reduces atherosclerosis in apolipoprotein E-deficient mice. Glycobiology 2013; 23: 654-663.

14 Henderson NC, Mackinnon AC, Farnworth SL, et al. Galectin-3 expression and secretion links macrophages to the promotion of renal fibrosis. Am J Pathol 2008; 172: 288-298.

15 Delaine T, Collins P, MacKinnon A, et al. Galectin-3-binding glycomimetics that strongly reduce bleomycin-induced lung fibrosis and modulate intracellular glycan recognition. Chembiochem 2016; 17: 1759-1770.

16 MacKinnon AC, Farnworth SL, Hodkinson PS, et al. Regulation of alternative macrophage activation by galectin-3. J Immunol 2008; 180: 2650-2658.

17 Mackinnon AC, Humphries D, Mills R, et al. Deletion of galectin-3 in myeloid cells inhibits bleomycin-induced lung fibrosis. Am J Respir Crit Care Med 2019; 199: A2422.

18 Parmar NR, Mackinnon AC, Zetterberg F, et al. Role of galectin-3 in idiopathic pulmonary fibrosis development. Am J Respir Crit Care Med 2019; 199: A5328.

19 Hirani N, Mackinnon A, Nicol L, et al. TD139, a novel inhaled galectin-3 inhibitor for the treatment of idiopathic pulmonary fibrosis (IPF). Results from the first in (IPF) patients study. Am J Respir Crit Care Med 2017; 195: A7560.

20 Klech H, Pohl W. Technical recommendations and guidelines for bronchoalveolar lavage (BAL). Report of the European Society of Pneumology Task Group. Eur Respir J 1989; 2: 561-585.

21 Naderer OJ, Rodvold KA, Jones LS, et al. Penetration of GSK1322322 into epithelial lining fluid and alveolar macrophages as determined by bronchoalveolar lavage. Antimicrob Agents Chemother 2014; 58: 419-423.

22 Rennard SI, Basset G, Lecossier D, et al. Estimation of volume of epithelial lining fluid recovered by lavage using urea as marker of dilution. J Appl Physiol 1986; 60: 532-538.

23 Molyneaux PL, Smith JJ, Saunders S, et al. BAL is safe and well tolerated in individuals with idiopathic pulmonary fibrosis: an analysis of the PROFILE study. Am J Respir Crit Care Med 2021; 203: 136-139.

24 Sakamoto K, Taniguchi H, Kondoh Y, et al. Acute exacerbation of IPF following diagnostic bronchoalveolar lavage procedures. Respir Med 2012; 106: 436-442.

25 Lukey PT, Harrison SA, Yang S, et al. A randomised, placebo-controlled study of omipalisib (PI3K/mTOR) in idiopathic pulmonary fibrosis. Eur Respir J 2019; 53: 1801992.

26 Maher TM, van der Aar EM, Van de Steen O, et al. Safety, tolerability, pharmacokinetics, and pharmacodynamics of GLPG1690, a novel autotaxin inhibitor, to treat idiopathic pulmonary fibrosis (FLORA): a phase 2a randomised placebo-controlled trial. Lancet Respir Med 2018; 6: 627-635.

27 Wells AU, Hansell DM, Harrison NK, et al. Clearance of inhaled 99mTc-DTPA predicts the clinical course of fibrosing alveolitis. Eur Respir J 1993; 6: 797-802.

28 Bonner JC. Regulation of PDGF and its receptors in fibrotic diseases. Cytokine Growth Factor Rev 2004; 15: 255-273.

29 Martinet Y, Rom WN, Grotendorst GR, et al. Exaggerated spontaneous release of platelet-derived growth factor by alveolar macrophages from patients with idiopathic pulmonary fibrosis. N Engl J Med 1987; 317: $202-209$.

30 Ziora D, Jastrzebski D, Adamek M, et al. Circulating concentration of markers of angiogenic activity in patients with sarcoidosis and idiopathic pulmonary fibrosis. BMC Pulm Med 2015; 15: 113.

31 Zhu X, Fang X, Chen W, et al. Lower expression of platelet derived growth factor is associated with better overall survival rate of patients with idiopathic nonspecific interstitial pneumonia. J Thorac Dis 2017; 9: 519-528.

32 Pechkovsky DV, Prasse A, Kollert F, et al. Alternatively activated alveolar macrophages in pulmonary fibrosis-mediator production and intracellular signal transduction. Clin Immunol 2010; 137: 89-101.

33 Prasse A, Pechkovsky DV, Toews GB, et al. A vicious circle of alveolar macrophages and fibroblasts perpetuates pulmonary fibrosis via CCL18. Am J Respir Crit Care Med 2006; 173: 781-792.

34 Flevaris P, Vaughan D. The role of plasminogen activator inhibitor type-1 in fibrosis. Semin Thromb Hemost 2017 43: 169-177.

35 Eitzman DT, McCoy RD, Zheng X, et al. Bleomycin-induced pulmonary fibrosis in transgenic mice that either lack or overexpress the murine plasminogen activator inhibitor-1 gene. J Clin Invest 1996; 97: 232-237.

36 Hattori N, Degen JL, Sisson TH, et al. Bleomycin-induced pulmonary fibrosis in fibrinogen-null mice. J Clin Invest 2000; 106: 1341-1350.

37 Menou A, Duitman J, Crestani B. The impaired proteases and anti-proteases balance in idiopathic pulmonary fibrosis. Matrix Biol 2018; 68-69: 382-403.

38 Kotani I, Sato A, Hayakawa H, et al. Increased procoagulant and antifibrinolytic activities in the lungs with idiopathic pulmonary fibrosis. Thromb Res 1995; 77: 493-504.

39 Korthagen NM, van Moorsel CHM, Zanen P, et al. Evaluation of circulating YKL-40 levels in idiopathic interstitial pneumonias. Lung 2014; 192: 975-980.

40 Korthagen NM, van Moorsel CHM, Barlo NP, et al. Serum and BALF YKL-40 levels are predictors of survival in idiopathic pulmonary fibrosis. Respir Med 2011; 105: 106-113.

41 Furuhashi K, Suda T, Nakamura Y, et al. Increased expression of YKL-40, a chitinase-like protein, in serum and lung of patients with idiopathic pulmonary fibrosis. Respir Med 2010; 104: 1204-1210.

42 Neighbors M, Cabanski CR, Ramalingam TR, et al. Prognostic and predictive biomarkers for patients with idiopathic pulmonary fibrosis treated with pirfenidone: post-hoc assessment of the CAPACITY and ASCEND trials. Lancet Respir Med 2018; 6: 615-626.

43 Adegunsoye A, Alqalyoobi S, Linderholm A, et al. Circulating plasma biomarkers of survival in anti-fibrotic treated patients with idiopathic pulmonary fibrosis. Chest 2020; 158: 1526-1534. 